\title{
Good chemistry between proteins and materials
}

\section{ce \\ This strategy enables \\ virtually any polyglycine- containing peptide to be appended quantitatively onto the \\ C-terminus of recombinant proteins}

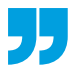

\section{Reversibly modifying 3D}

biomaterials with active proteins would greatly improve the control of cell function in vitro. Attaching full-length proteins to materials, such as hydrogels, requires the introduction of chemical groups in the protein structure that react with the material without compromising protein activity. Now, writing in Nature Materials, Cole DeForest and colleagues report a site-specific chemoenzymatic protein modification strategy that maintains protein activity and, in combination with photochemistry, allows the spatially and temporally controlled photopatterning of hydrogels in 3D.

In vivo, cells dynamically interact with the proteins in their microenvironment, which guide and direct cell fate and thus are key to cellular function. DeForest and colleagues recreated this complex

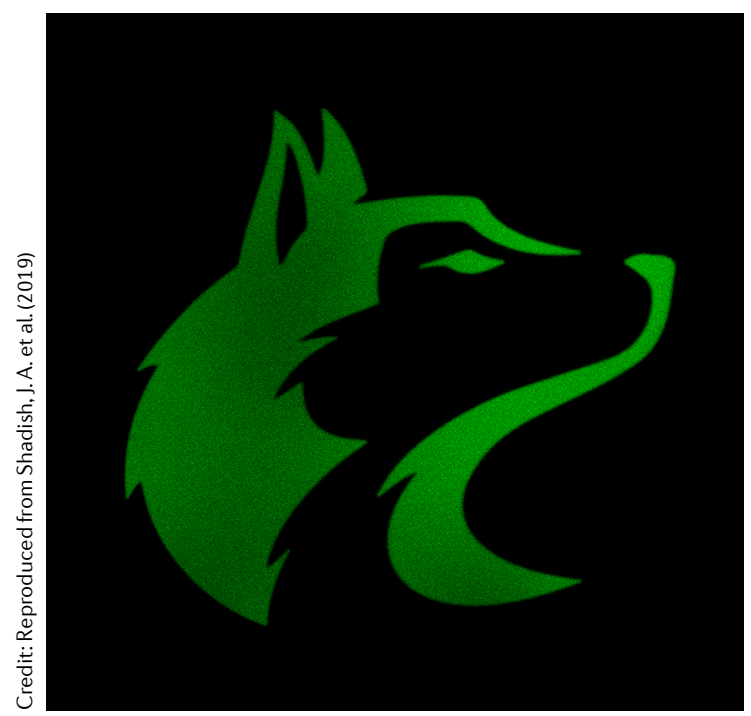

interplay in cytocompatible 3D hydrogels by embedding cells within a hydrogel spatially decorated with specific active proteins. Previously, attaching full-length proteins to hydrogels has relied on the interaction of cysteines and lysines with amine-reactive and sulfhydrylreactive compounds, which leads to unspecific, often non-reproducible modification and substantial loss of protein activity. Moreover, on-demand control of protein binding and unbinding in a hydrogel remains challenging.

DeForest and colleagues use a chemoenzymatic strategy to site-specifically functionalize proteins with bioorthogonal reactive groups. By fusing proteins to the bacterial transpeptidase sortase, which recognizes a specific sorting signal appended to the protein, a tag can be conjugated to the protein through subsequent nucleophilic displacement of the enzyme with a polyglycine peptide. "This strategy enables virtually any polyglycine-containing peptide to be appended quantitatively onto the C-terminus of recombinant proteins - a feature that we have exploited to install several different bioorthogonal reactive groups site-specifically onto many proteins of interest," explains DeForest.

The researchers tagged fluorescent proteins, growth factors and enzymes with different polyglycine probes featuring reactive groups such as azides and aromatic aldehydes. These functional groups facilitate covalent attachment of the proteins to hydrogels through click chemistry. Importantly, the tagged proteins maintain their bioactivity in the hydrogel. By using hydrogels with photocaged reactive groups, mask-based photolithography can be applied to spatially and temporally control protein binding using light. Similarly, by tethering reactive species amenable to photoscission, the proteins can also be released from the hydrogel. "Taking advantage of the patterning precision that photochemistry affords in conjunction with these engineered proteins, we gain the unique ability to govern $4 \mathrm{D}$ cell fate with subcellular spatial resolution," says DeForest.

Using this strategy, DeForest and colleagues demonstrated that specific cellular signalling pathways can be switched on by patterning hydrogels with active growth factors. Moreover, the possibility to reversibly control protein-hydrogel binding by light enables the on-demand modulation of cell proliferation and migration in specific regions of the hydrogel.

Being able to control 4D presentation of active growth factors and other fragile proteins opens up the door to spatially control stem cell differentiation and adult cell function within biomaterials. "We are excited to use this technology to better understand dynamic cellmatrix interactions and to engineer heterogeneous multicellular tissues," comments DeForest.

Christine-Maria Horejs

ORIGINAL ARTICLE Shadish, J.A. et al. Bioactive site-specifically modified proteins for $4 \mathrm{D}$ patterning of gel biomaterials. Nat. Mater. https://doi.org/ 10.1038/s41563-019-0367-7 (2019) 